\title{
Dinamiche della bellezza: sguardo e narrazione nel ritratto
}

\author{
di Silvana Borutti
}

silvana.borutti@unipv.it

\begin{abstract}
Through the commentary on portraits (Lotto, Giorgione, Vermeer, L. Freud, Rembrandt, Tiziano, Bacon...), my contribution will develop two topics. In fact, the portrait calls to reflect both on the reversible dynamics of the gaze (between painter, subject and spectator, between presence and absence) and on the time of the individual as an experience and as a transformative process that tells a story.
\end{abstract}

Keywords: Portrait, Dynamics of the Gaze, Time of the Individual, Telling a Story

\section{Il ritratto e lo sguardo}

Lo sguardo è forse per l'uomo l'esperienza estetica più complessa. Con la sua prospettiva fenomenologica, Maurice Merleau-Ponty ci ha insegnato che lo sguardo umano mette in campo dinamiche di reversibilità e di scambio, che possono essere meglio comprese se si considera il carattere esemplare dello sguardo in pittura, e in particolare il gioco o la danza degli sguardi tra pittore, immagine, spettatore - una danza che diventa ancora più complessa se si analizzano quelle immagini che sono i ritratti. Rifletteremo dunque prima sullo sguardo come esperienza percettiva speciale, capace di mettersi in relazione con quell'evento del campo visivo che è una rappresentazione pittorica.

Ma in primo luogo, e in generale, che esperienza è il guardare? Che cosa sia il guardare come parte dell'essere, lo dicono i neurofisiologi e gli psicologi della percezione. La prospettiva filosofico-fenomenologica può invece dire qualcosa su ciò che qualifica l'esperienza del guardare come evento dell'essere 
al mondo. Il guardare non è pura impressione passiva, un semplice incontro percettivo col dato, un incontro di un soggetto e un oggetto contrapposti. Scrive Wittgenstein:

L'occhio umano noi non lo vediamo come un ricettore, l'occhio non sembra lasciar entrare qualcosa, bensì sembra che lo emetta. L'orecchio riceve; l'occhio guarda. (Lancia sguardi, s'accende di lampi, è raggiante, risplende). Con l'occhio si può atterrire, ma non con l'orecchio o col naso. Se vedi l'occhio, vedi qualcosa che ne esce. Vedi lo sguardo dell'occhio ${ }^{1}$.

Il guardare non è un ricevere passivo, ma è un'esperienza, un atto che avviene in un campo e in un orizzonte di esperienza. Guardando, siamo immersi nel mondo attraverso il corpo proprio, siamo perciò già integrati in un orizzonte di senso, in una fisionomia, in un mondo, siamo già in rapporto con gli altri che hanno visto prima di noi e che vedono con no $i^{2}$. La visione sensibile è già abitata da un senso. Merleau-Ponty dice che non vediamo transitivamente un questo (voir ceci) ma vediamo secondo (voir selon) ${ }^{3}$ : vediamo il mondo secondo la curvatura, il campo sensibile aperto da ciò che stiamo guardando - guardiamo non la cosa o l'immagine, ma l'apparire, l'apparizione, il fenomeno di quella cosa con le sue risonanze. Quando guardiamo la rosa, guardiamo «la roseità che si estende attraverso la rosa», guardiamo il mondo attraverso la rosa ${ }^{4}$ - in uno sconfinamento e intreccio (entrelac) tra visione e visibilità, tra vedente e visibile. Il nostro vedere diventa così sguardo.

E in pittura? Poiché dunque "guardare semplicemente" e passivamente non si dà, non ci è possibile affondarci semplicemente, immediatamente, nella visione di un quadro. Nelson Goodman richiama l'attenzione sull'esperienza

\footnotetext{
${ }^{1}$ L. Wittgenstein, Osservazioni sulla filosofia della psicologia (1980), tr. it. a cura di R. De Monticelli, Adelphi, Milano 1990, I, § 1100.

${ }^{2}$ Husserl parla di Mitwahrnehmung, co-percezione. E. Husserl, Meditazioni cartesiane (1950), tr. it. a cura di F. Costa, Bompiani, Milano 1960, p. 141.

${ }^{3}$ Cfr. J. Garelli, "Voir ceci et voir selon", in M. Richir e. Tassin (éd. par), Merleau-Ponty. Phénoménologie et expériences, Millon, Grenoble 1992, pp. 79-99.

${ }_{4} \mathrm{M}$. Merleau-Ponty, Il visibile e l'invisibile (1964), nuova ed. it. a cura di M. Carbone, Bompiani, Milano 1993, p. 192.
} 
per cui comincio a guardare diversamente due quadri che mi sono sembrati fino a quel momento identici, a partire dal momento in cui mi viene detto che uno è l'originale e l'altro la copia, e che a un certo punto avrò imparato a vederne la differenza "estetica" - nel senso della differenza non solo e non tanto delle proprietà percepibili dei quadri, ma del modo in cui devono essere guardati5. Se vedo per la prima volta un Vermeer e una copia di van Meegeren, è facile che sia ingannata; ma sarà sempre meno facile che mi sbagli dopo che avrò visto tanti Vermeer. Vedere tanti Vermeer, significa imparare a vedere la deviazione, la deformazione configurante che la pittura di Vermeer impone al visibile ${ }^{6}$. In questo senso, lo sguardo non è un'impressione istantanea, ma è un'esperienza dinamica e processuale

Esemplare la dinamicità, la danza degli sguardi che si crea attraverso quell'evento nel campo visivo che è l'immagine pittorica. Il quadro offre «le tracce della visione dell'interno» ${ }^{7}$ del pittore - di ciò che si è visto in lui; offre la forza dello sguardo del pittore a chi guarda: lo sguardo del pittore è in fondo ciò che fa comparire il quadro come un'apparizione, è un gesto che fa emergere dall'invisibile e dall'assenza la figura come dono di visibilità. Il visibile non è allora ciò che vedo, ma è un evento di visibilità: la pittura mette in scena il vedere, e le sue deformazioni. Emilio Tadini fa notare in un quadro di Caravaggio uno strano sguardo che sembra avere a che fare con la luce stessa in sé - tanto che questo sguardo appare come, diremmo quasi, uno sguardo al quadrato, una percezione della percezione.

\footnotetext{
${ }^{5}$ N. Goodman, I linguaggi dell'arte (1968), tr. it. a cura di F. Brioschi, il Saggiatore, Milano 1976, pp. 98-99.

${ }^{6}$ Cfr. M. Merleau-Ponty, La prosa del mondo (1969), tr. it. a cura di C. Sini, Editori Riuniti, Roma 1984, p. 86.

${ }^{7}$ M. Merleau-Ponty, L'occhio e lo spirito (1964), tr. it. di A. Sordini, SE, Milano 1989, p. 22.
} 


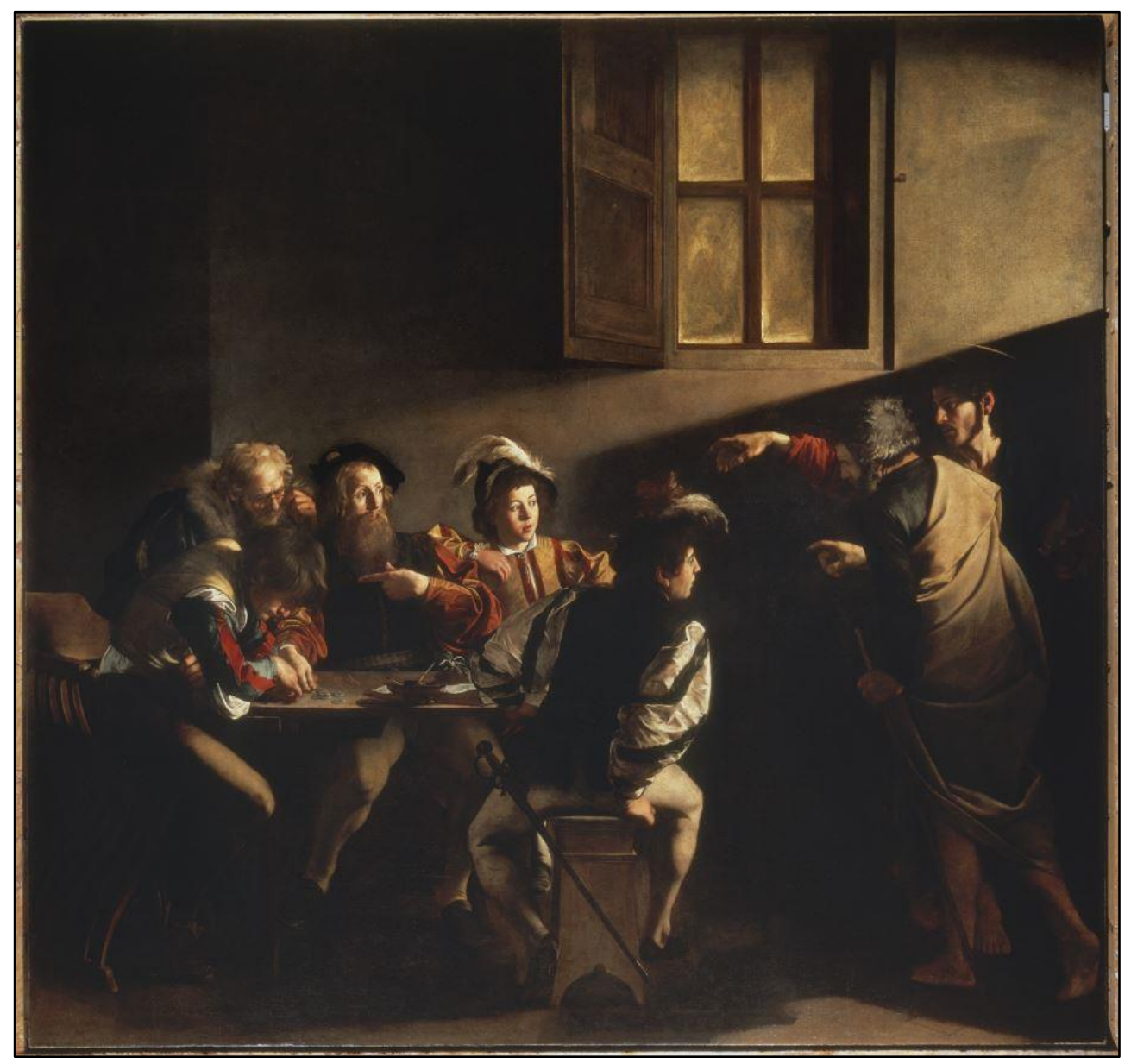

Figura 1: Caravaggio, Vocazione di San Matteo, 1599-1610

Cappella in San Luigi dei Francesi, Roma.

Dove guardano? San Matteo e i compagni al tavolo non guardano l'apparizione di Cristo (dovrebbero avere la testa di profilo, per poter guardare Cristo contro la parete), ma piuttosto la luce che colpisce il dorso di Pietro e il volto di Cristo. Guardano la luce come fonte del visibile, guardano l'interiorità dell'apparizione, la figura, la visibilità, il mostrarsi ${ }^{8}$. I personaggi guardano la luce della pittura, ciò che porta a visibilità.

Di un tema analogo parla Heidegger nell'Origine dell'opera d'arte quando scrive del tratto, Riss, in pittura. Nel segno pittorico, nel "tratto" che realizza lo sguardo del pittore, Heidegger vede la lotta tra illuminazione e

8 Cfr. E. Tadini, "Il santo, il dialogo", in G. Damiani (a cura di), L’immagine. Arte, scienza, teoria, Carlini, Milano 1989, pp. 205-214. 
nascondimento, tra visibile e invisibile da cui scaturisce, attraverso il dinamismo della mano, il contorno della figura9. Il tratto heideggeriano espone all'esistenza, ritma e realizza lo sguardo del pittore.

Il quadro si enuncia come apertura-recinzione di uno spazio di visibilità: come nel linguaggio l'enunciazione è l'atto che fa emergere una cosa detta e significante dal silenzio, così lo sguardo fa emergere una figura dall'invisibilità. L'immagine continua sempre di nuovo a costruirsi e a imporsi autoriflessivamente, attraverso l'efficacia topologica della cornice e del titolo, secondo un movimento di esclusione-inclusione ${ }^{10}$. Scrive il pittore Ruggero Savinio: «La cornice [...] apre lo spazio della figura e chiude l'infinito spazio avvolgente, l'apeiron, che la circonda da ogni parte. La cornice comunica col fuori e col dentro, è un confine fragile, tremante, oscillante» ${ }^{11}$. La cornice fa emergere la forma che attira il nostro sguardo. Il nostro sguardo risponde così all'effetto autoassertivo del quadro come ritaglio nel visibile, si espone alla forza dell'immagine che si enuncia nel quadro ("c'è qualcosa da vedere"). Attirando il nostro sguardo, l'immagine si fa soggetto di sguardo, in altre parole, non subisce, ma agisce.

Ora, poiché il nostro tema è il ritratto, dobbiamo chiederci come il ritratto sia soggetto di sguardo. Che sguardo è quello del ritratto? Non è uno sguardo che veda oggetti, è semmai la presenza dello sguardo, che mette in gioco, espone un soggetto autonomo, un soggetto considerato di per sé. Un bel ritratto è un ritratto da cui emerga un "per sé" presente in una determinata forma di assenza $a^{12}$, presente non perché somigliante al modello, presente cioè

\footnotetext{
9 «La lotta non è un tratto che spalanchi un baratro, ma è l'intimità di un convenirsi reciproco dei lottanti [...] La lotta che viene condotta nel tratto [...] è l'origine della figura». M. Heidegger, "L'origine dell'opera d'arte", in Sentieri interrotti (1950), tr. it. a cura di P. Chiodi, La nuova Italia, Firenze 1968, pp. pp. 3-69, qui pp. 46-47.

${ }_{10}$ Cfr. F. Petrella, "Quadro e cornice: il setting clinico", in F. Petrella, Turbamenti affettivi e alterazione dell'esperienza, Raffaello Cortina, Milano 1993, pp. 127-141.

${ }^{11}$ R. Savinio, "La figura e l'altro", Quaderni Warburg Italia, I, 2003, pp. 319-328, qui p. 321.

12 Sulla forma di presenza del soggetto del ritratto, cfr. J.-L. Nancy, "Il ritratto autonomo", in J.-L. Nancy, Il ritratto e il suo sguardo (2000), tr. it. a cura di R. Kirchmayr, Raffaello Cortina, Milano 2002, pp. 11-28.
} 
non in modo anagrafico, ma perché, nell'atto dell'esporsi, espone un'interiorità, un "per sé" - ciò che siamo abituati a chiamare "anima". Come scrive Leonardo nel Trattato della pittura:

290. Degli atti delle figure.

Farai le figure in tale atto, il quale sia sufficiente a dimostrare quello che la figura ha nell'animo; altrimenti la tua arte non sarà laudabile ${ }^{13}$.

Guardiamo allora al ritratto come esposizione di un per sé. Nancy commenta il Ritratto di giovane detto alla lampada di Lotto: scrive che lo sguardo del personaggio si traspone nello sguardo della pittura stessa. La pittura diventa sguardo, o sguardo dello sguardo, sguardo al quadrato ${ }^{14}$. Il ritratto come soggetto di sguardo ${ }^{15}$.

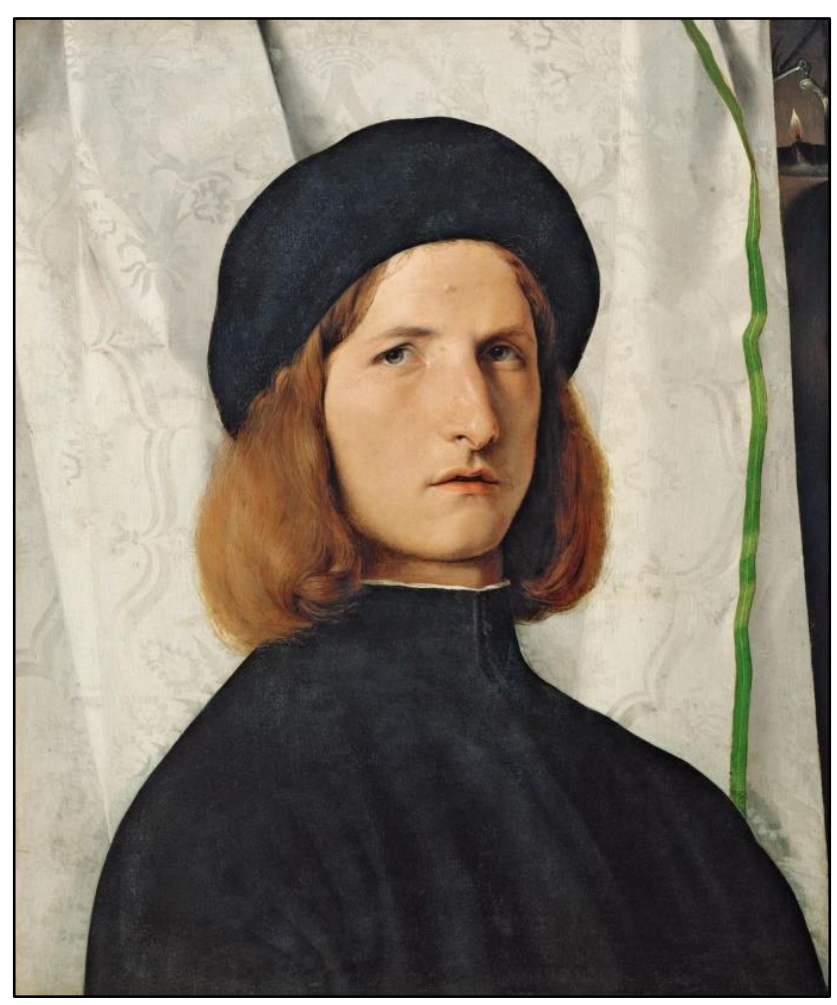

Figura 2: Lorenzo Lotto, Ritratto di giovane detto "alla lampada", 1506-1507, Kunsthistorisches Museum, Wien.

${ }^{13}$ Leonardo da Vinci, Trattato della pittura, $§ 290$ [on-line]. Edizione digitale a cura di A. Borzelli, tratta da "Trattato della pittura" di Leonardo da Vinci, Carabba Editore, Lanciano 1947. Pubblicato in data 17 febbraio 2016, consultato in data 15 novembre 2021. Disponibile all'indirizzo: https:/www.liberliber.it/mediateca/libri//leonardo/trattato_della pittura/html/index.htm. 14 J.-L. Nancy, Il ritratto e il suo sguardo, cit., pp. 65 sgg.

${ }^{15}$ L. Lavaud, L’image, Flammarion, Paris 1999, p. 11. 
Ci colpisce il movimento della tenda damascata e luminosa da cui il giovane sembra emergere, come se ne venisse portato a visibilità: una presenza che viene dall'assenza.

Il titolo del quadro ci richiama a quel punto luce che si vede a destra, la lampada, che raddoppia lo sguardo del dipinto. Ora, la presenza della lampada non è un caso. Si trovano molti dispositivi ottici nei ritratti, che alludono alla luce, all'irraggiarsi di uno sguardo: punti luce che lanciano sguardi. Vengono certamente alla mente gli specchi nella pittura fiamminga, che raddoppiano la visibilità e la presenza dei personaggi:

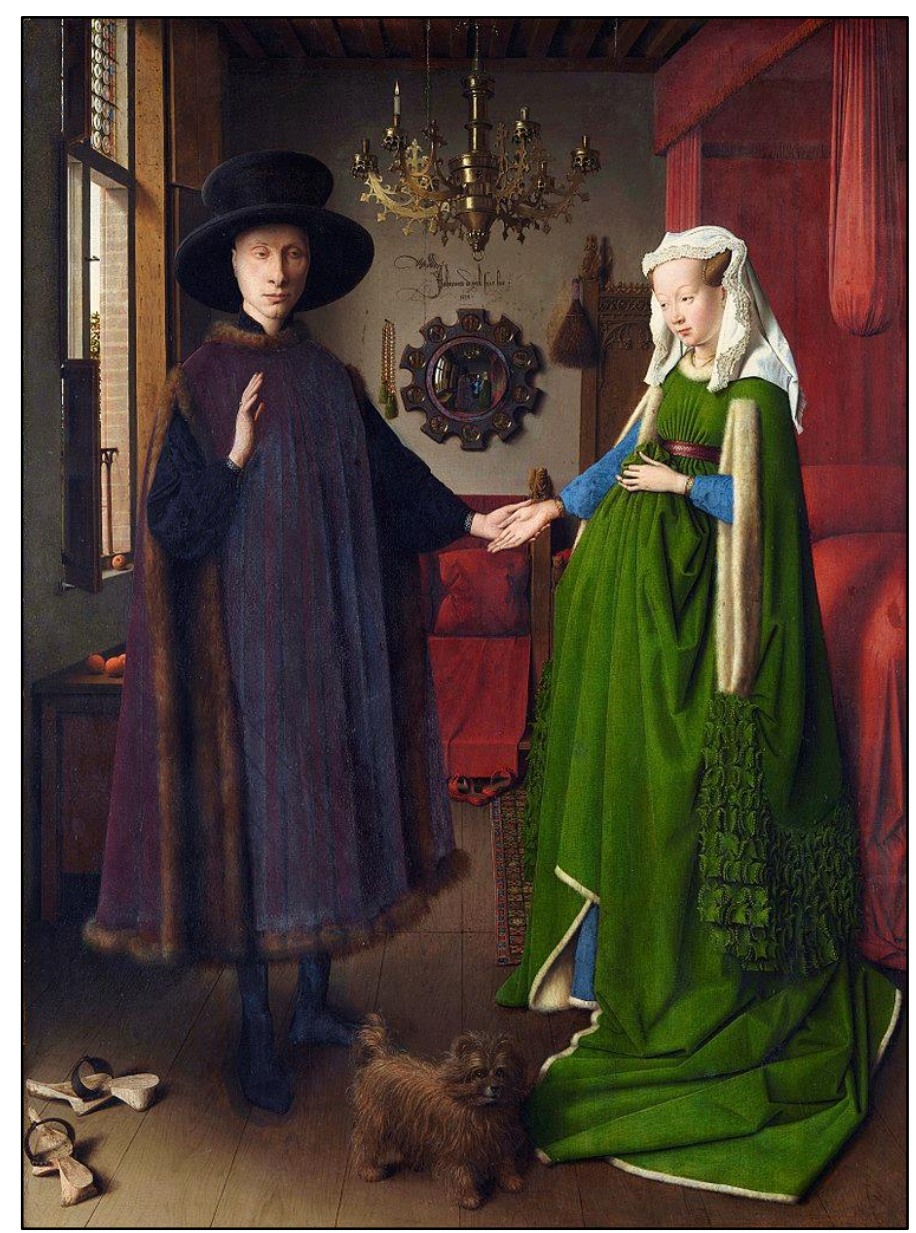

Figura 3: Jan Van Eyck, Ritratto dei coniugi Arnolfini, 1434, National Gallery, London. 
$\mathrm{O}$, ancora, il dispositivo anamorfico che, da una certa angolatura, ci fa vedere il teschio negli Ambasciatori di Holbein. Ma possiamo pensare ad altri dispositivi ottici, come la perla che risplende sensualmente con gli occhi e con le labbra della Ragazza col turbante di Vermeer.

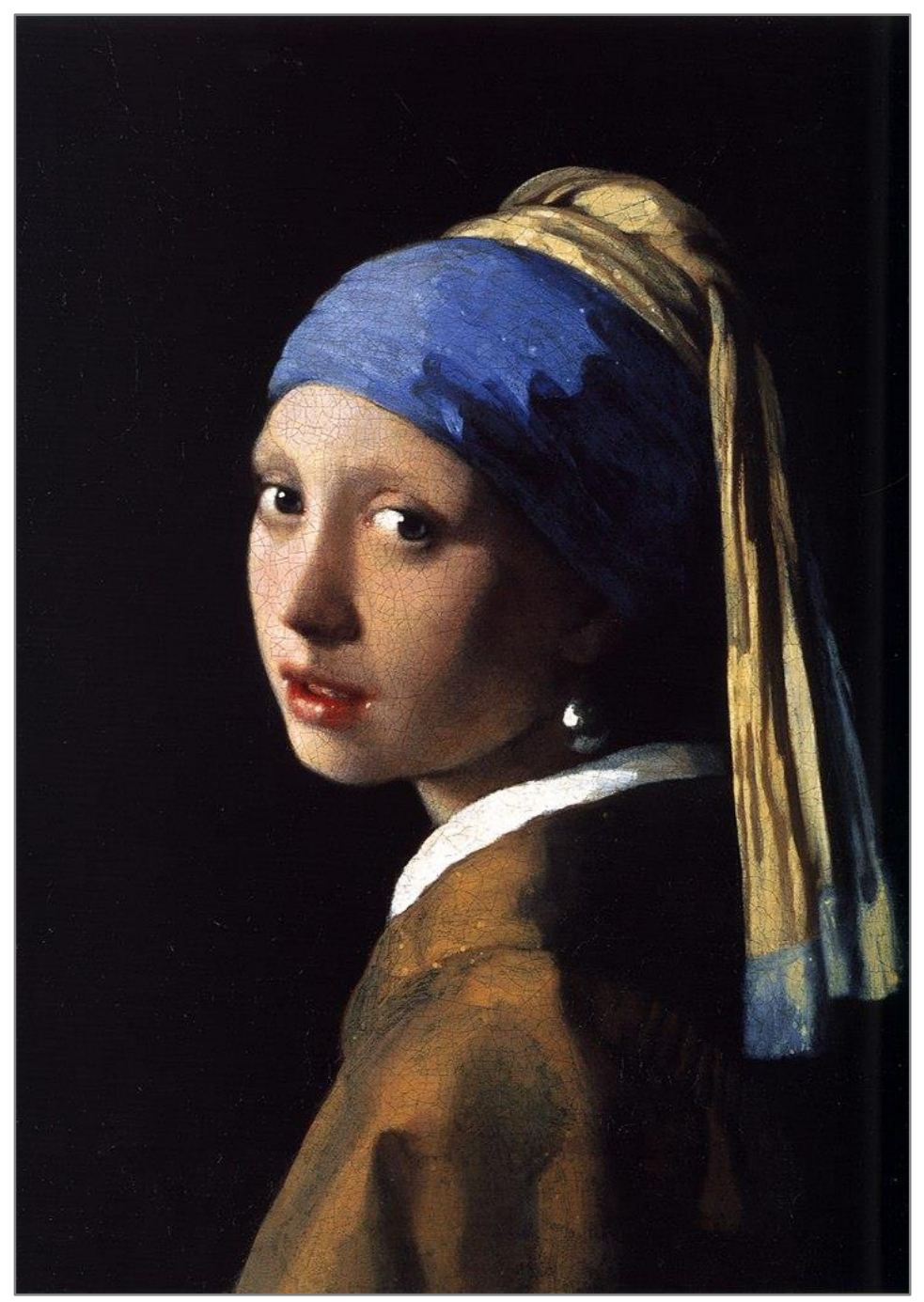

Figura 4: Johannes Vermeer, Ragazza col turbante, 1665-1666, Mauritshuis, Den Haag. 
O anche alle perle della corona e alle orecchie della Regina Elisabetta nel ritratto di Lucian Freud, che richiamano l'attenzione sullo sguardo severo e rassegnato al comando.

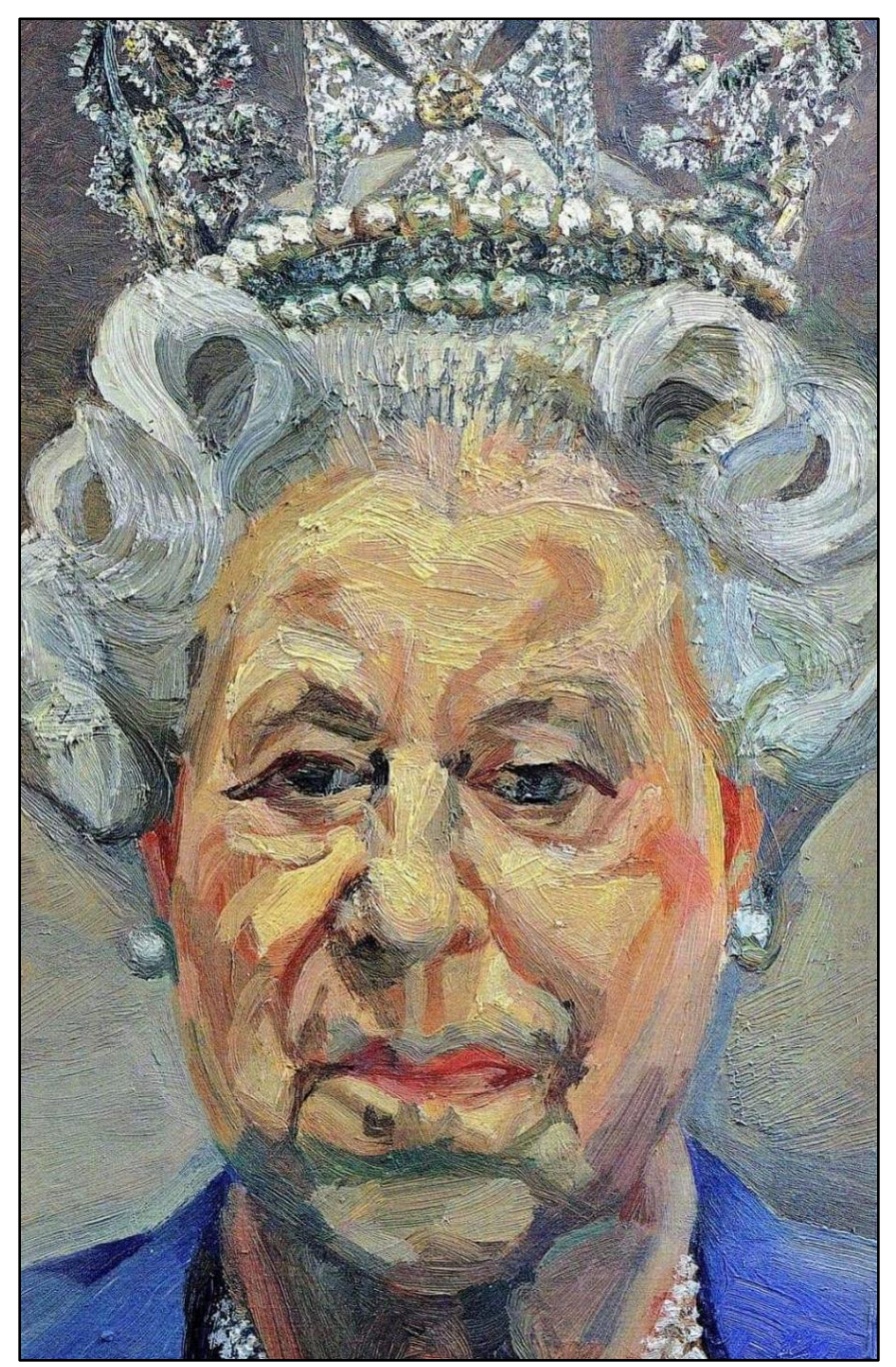

Figura 5: Lucian Freud, Sua Maestà la Regina Elisabetta II, 2000-2001, Collezione Reale. 
Si capisce dal vagare del nostro sguardo sui ritratti che lo sguardo non è solo degli occhi, è di tutto il quadro, è il quadro che si organizza intorno allo sguardo. Come scrive Wittgenstein nelle Osservazioni sui colori:

Un colore 'riluce' in un ambiente (come gli occhi sorridono soltanto in un volto' ${ }^{16}$.

E nelle Ricerche filosofiche:

Una bocca sorridente sorride soltanto in un volto umano ${ }^{17}$.

Tutta la figura e la composizione fanno lo sguardo del quadro. Così è da tutta la composizione che emergono i due diversi sguardi nel Doppio ritratto di Giorgione.

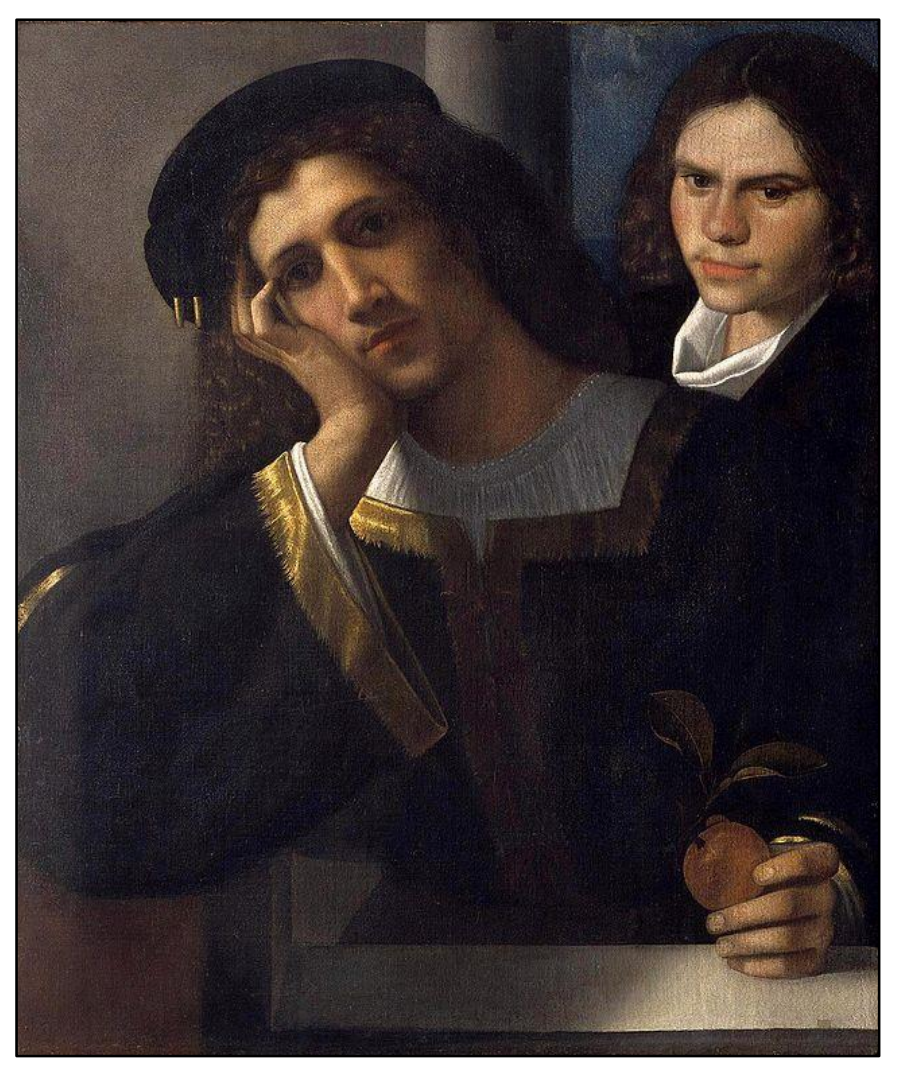

Figura 6: Giorgione, Doppio ritratto, 1502 circa, Palazzo Venezia, Roma.

${ }^{16}$ L. Wittgenstein, Osservazioni sui colori (1977), tr. it. di M. Trinchero, Introduzione di A. G. Gargani, Einaudi, Torino 1982, I, § 55 .

${ }_{17}$ L. Wittgenstein, Ricerche filosofiche (1953), tr. it. e cura di M. Trinchero, Einaudi, Torino 1967, ristampa 1974, I, § 583. 
Lo sguardo del quadro si raddoppia per il contrasto tra lo sguardo malinconico e innamorato del giovane in primo piano, e lo sguardo realistico, pragmatico dell'altro. Lo sguardo dell'innamorato (che tiene in mano una melagrana, simbolo dell'amore) emerge dalla luce radente della finestra; egli volge lo sguardo verso di noi, ma non ci vede, è perduto in sé. Nel catalogo della mostra Il volto e l'anima, da lui curata a Milano, Palazzo Reale, nel 1998-1999, Flavio Caroli scrive: «Si tratta di luce intellettuale, ben distinta da quella naturale esterna» ${ }^{18}$. La luce ridiventa naturale e cruda investendo l'altro volto, psicologicamente del tutto realistico.

Lo sguardo emerge dai volti ritratti come un'energia: questo intende Francis Bacon quando parla, per i suoi ritratti, di "emanazione”. Bacon ritrae in assenza dei modelli, poiché vuole essere libero di devastarne i lineamenti, e di farli emergere per distorsione e variazione. Egli dice:

Spero sempre di deformare le persone fino all'apparizione [I'm always hoping to deform people into appearance $]^{19}$.

Deformarle fino all'epifania: fino a far apparire l'emanazione della loro singolarità. Questi ritratti sono come oggetti tanto più reali e somiglianti, in quanto sopravvissuti alla deformazione e alla distruzione. L'immagine appare lavorata da un'istanza sfigurante; e il senso d'insieme si mostra in modo inaspettato, come un'emanazione, appunto, una specie di irradiamento personale, una forza interna depositata su un elemento esterno, come l'occhio della Rawsthorne (cfr. anche la foto su cui il pittore ha lavorato) o la fronte di Dyer.

${ }_{18}$ F. Caroli, "I moti dell'animo", in F. Caroli, L'anima e il volto. Ritratto e fisiognomica da Leonardo a Bacon, Electa, Milano 1998, p. 74.

${ }^{19}$ D. Sylvester, Interviews with Francis Bacon, Thames and Hudson, London 1993, p. 146. 


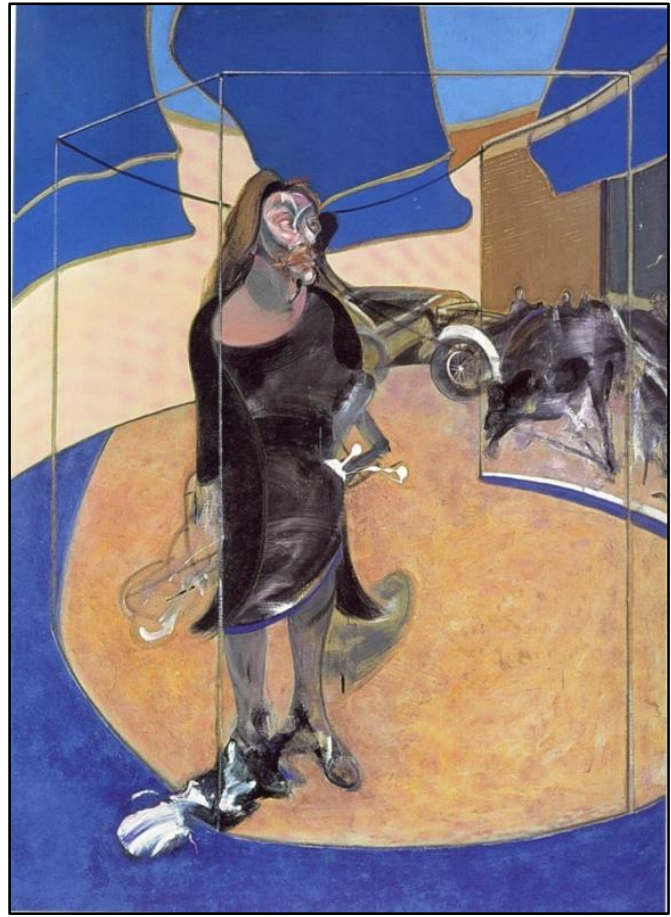

Figura 7: Francis Bacon, Ritratto di Isabel Rawsthorne in una via di Soho, 1967, Staatliche Museum, Berlin.

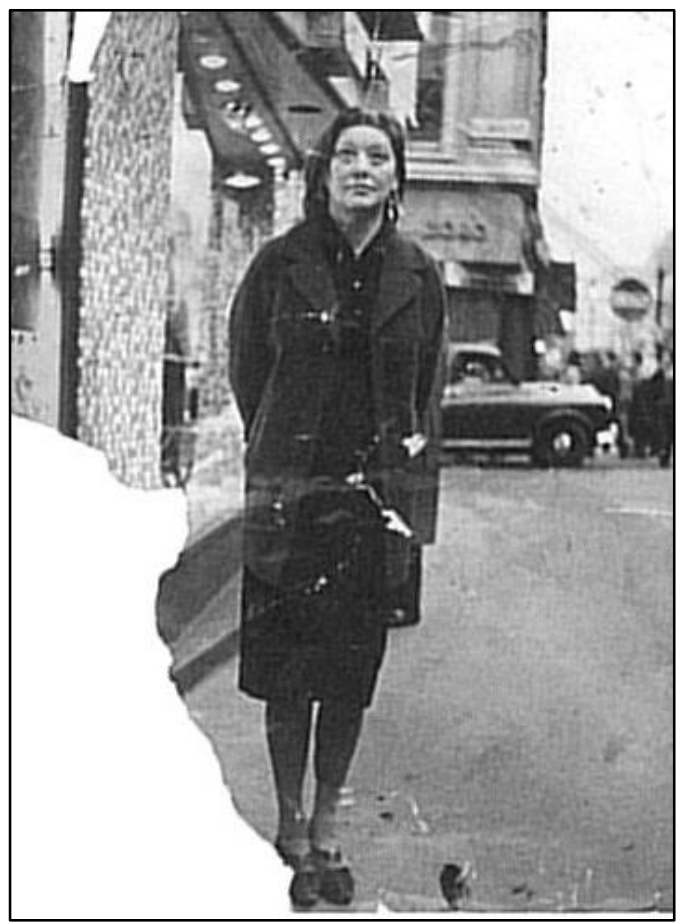

Figura 8: John Deakin, Fotografia di Isabel Rawsthorne in una via di Soho, 1967, Dublin City Gallery.

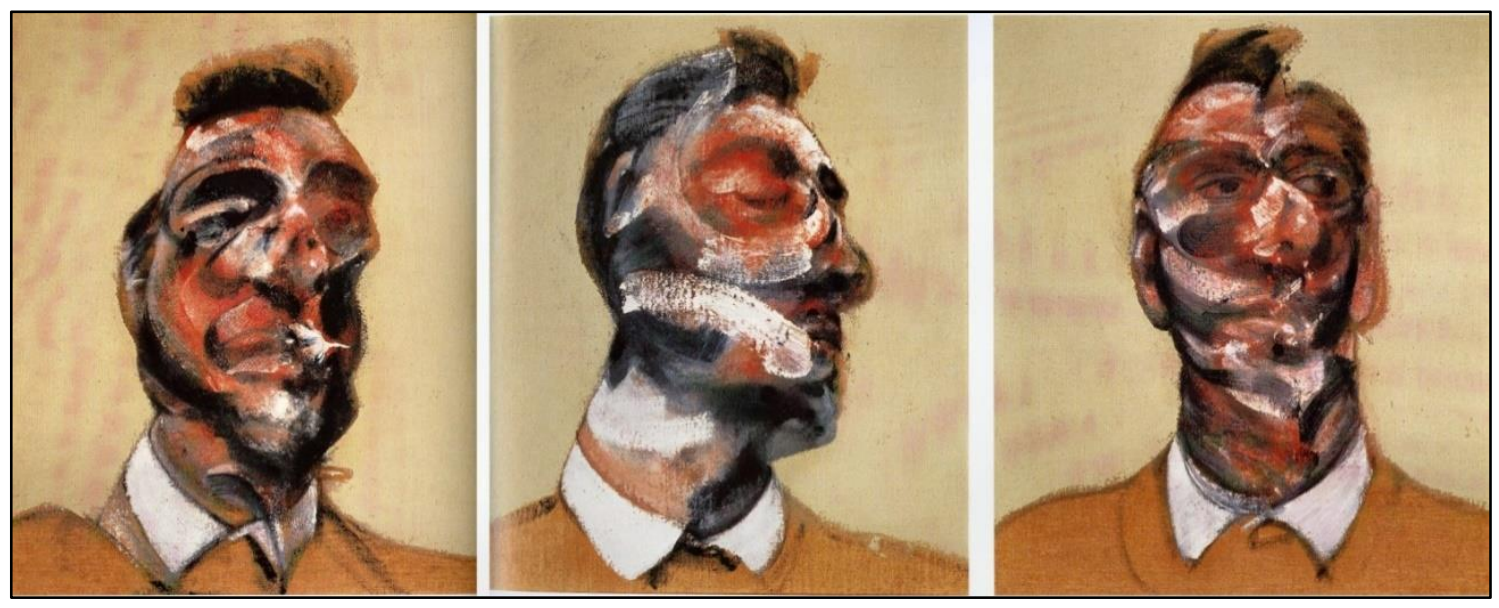

Figura 9: Francis Bacon, Tre studi per il ritratto di George Dyer, 1964, Collezione privata. 
Nei volti sfigurati di Bacon diventa chiaro che la somiglianza non è nel rapporto di vicinanza-distanza col modello, ma nell'autoasserzione del ritratto come individualità. La somiglianza viene alla luce, è un'apparizione: l'identità del ritratto è nel ritratto, è figura e sguardo.

E nel ritratto di Arthur Rimbaud di Alberto Giacometti, l'identità è nello sguardo che viene avanti dall'insieme occhi-bocca e emerge-sbuca dai tratti indefiniti del disegno, benché il soggetto sembri sottrarsi e cercare l'annichilimento. Giacometti scava ossessivamente e non rappresentativamente in ciò che vede: «Cerco la somiglianza assoluta e non l'apparenza» ${ }^{20}$. Si crea una somiglianza non mimetica, intrapsichica, che l'artista vede e fa vedere.

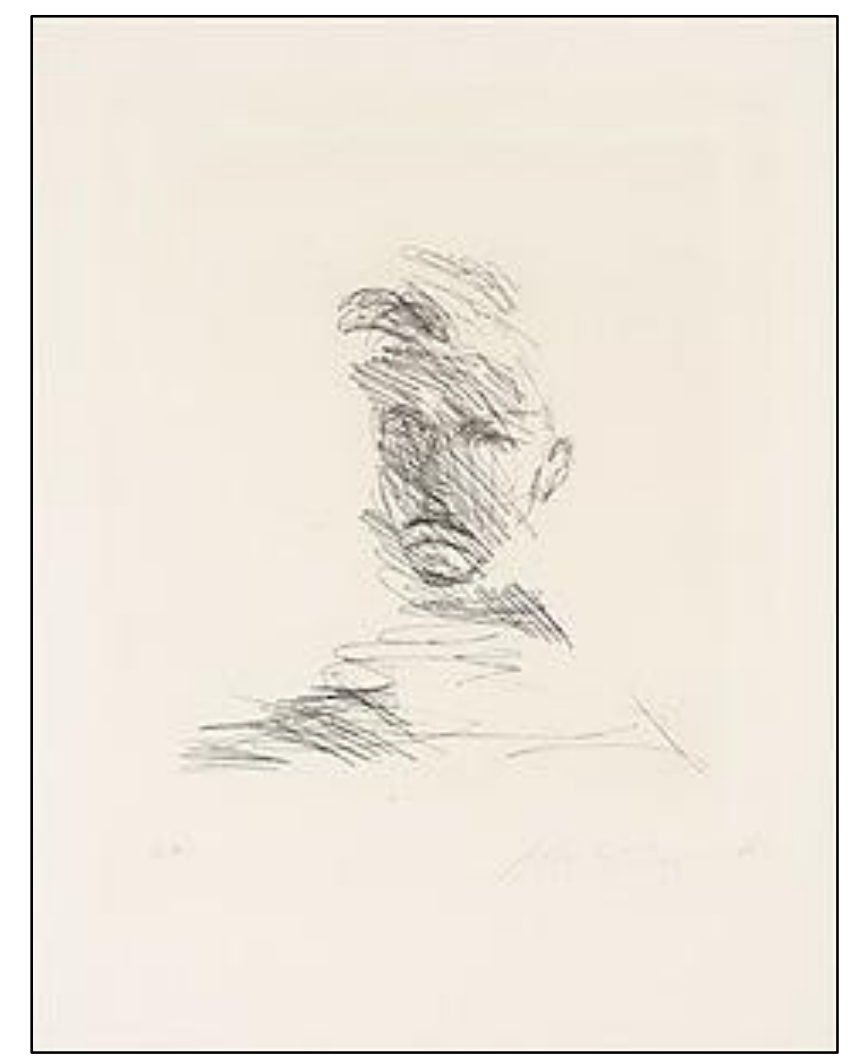

Figura 10: Alberto Giacometti, Ritratto di Rimbaud, 1962, Musée Rimbaud, Charleville.

20 J.-L. Daval, "Fou de réalité. Alberto Giacometti”, Journal de Genève, 8 giugno 1963, citato in J. Soldini, Alberto Giacometti. La somiglianza introvabile, Jaca Book, Milano 1998, p. 163. 
Lo storico dell'arte Alberto Martini scrive che dall'accanimento con cui Giacometti cerca di essere fedele alla propria percezione visiva - l'ossessiva fedeltà alla visione, per cui il pittore cerca una «rigorosa corrispondenza tra campo visivo reale e immagine dipinta» - «un'apparizione nasce magicamente e fora lo spazio bidimensionale per organizzarsi in una profondità lontana» ${ }^{21}$. L’identità del ritratto dunque non è quella anagrafica, ma il presentarsi assoluto del singolo individuo.

Le immagini come soggetto di sguardo sono anche sguardi che ci inquietano. Rinvio al commento che Roberto Calasso dedica all'inquietante gioco di sguardi e di sottrazione dello sguardo nel quadro di Degas La famiglia Bellelli22:

Il padre dà le spalle al pittore e le quattro figure guardano in direzioni diverse. Ciascuna sembra escludere le altre dal proprio campo visivo [...] Sono entità psichiche decise a non sfiorarsi. La madre ha uno sguardo così fisso e assente da poter sembrare cieca. Le due bambine sono recalcitranti: quella più vicina al centro distoglie lo sguardo dal pittore con determinazione dispettosa [...] L'altra fissa il pittore attediata, come dicesse: «Quando finirà questo tormento?» Il padre ignora il pittore - e soprattutto non ha sguardo ${ }^{23}$.

Ogni sguardo è qui sguardo assoluto e autonomo: sguardi che «non si incontrano e neppure si cercano» ${ }^{24}$, a significare l'infelicità di tutti.

\footnotetext{
${ }^{21}$ A. Martini, Alberto Giacometti, in I maestri del colore, n. 79, Fratelli Fabbri, Milano 1965.

22 R. Calasso, La folie Baudelaire, Adelphi, Milano 2008, pp. 214-217.

${ }^{23}$ Ivi, pp. 215-216.

${ }^{24}$ J.-L. Nancy, Il ritratto e il suo sguardo, cit., p. 18.
} 


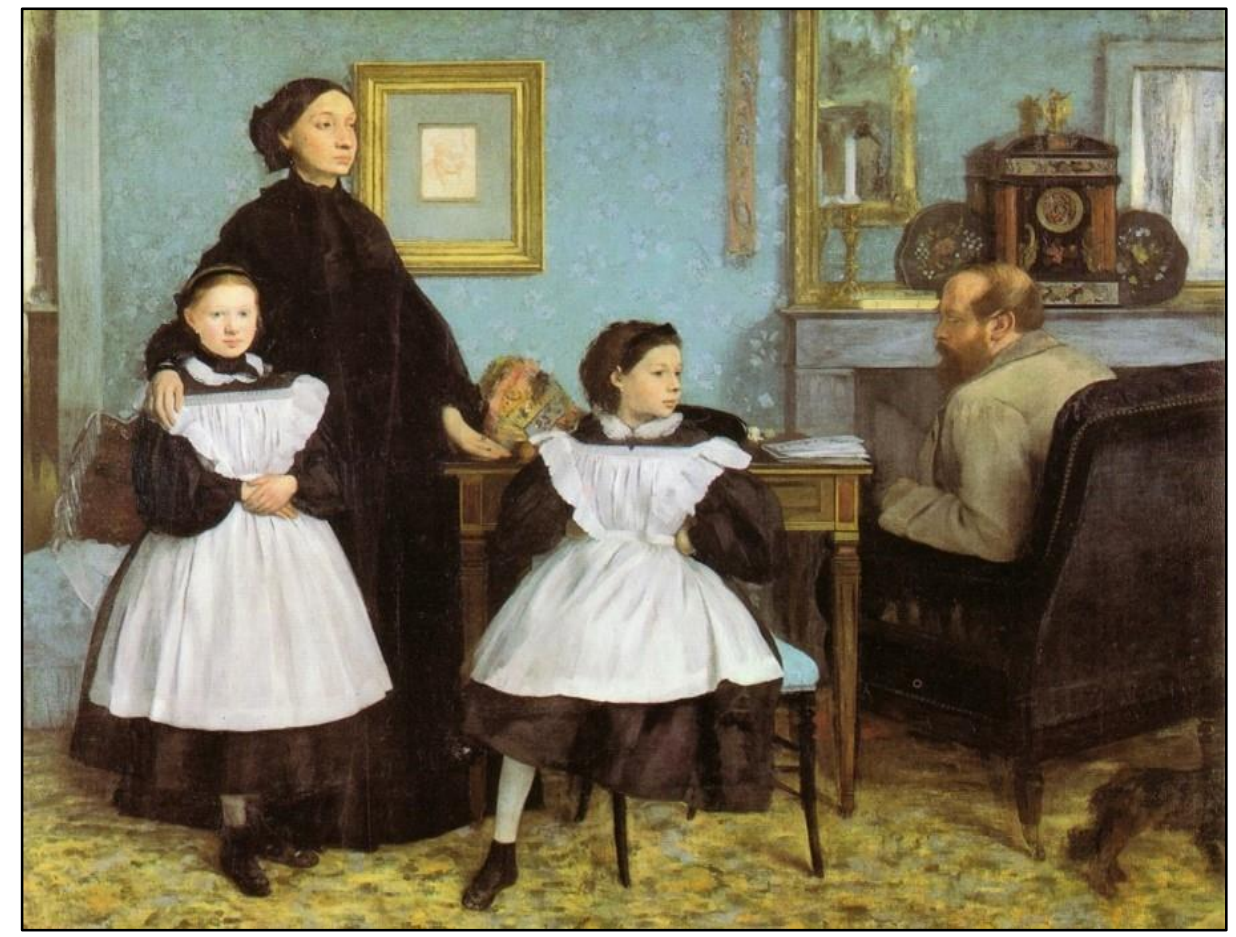

Figura 11: Edgar Degas, La famiglia Bellelli, 1858-1869, Musée d’Orsay, Paris.

\section{Il ritratto e la narrazione del tempo}

Il ritratto è dinamismo dello sguardo, ma è anche pensiero del tempo dell'individuo come esperienza e come processo trasformativo.

Possiamo cominciare banalmente dal topos ben noto delle tre età dell'uomo nell'Allegoria della prudenza di Tiziano. Il significato allegorico e moraleggiante sembra qui predominante: sopra il ritratto Tiziano ha scritto il motto che spiega il senso dell'allegoria: EX PRAETERITO / PRAESENS PRVDENTER AGIT / NI FVTVRA(M) ACTIONE(M) DETVRPET (Sulla base del passato / il presente prudentemente agisce / per non guastare l'azione futura). I tre animali sono il cane, simbolo della spensieratezza giovanile, il leone, simbolo della forza con la quale va affrontato il presente, il lupo, simbolo dei ricordi del passato. 


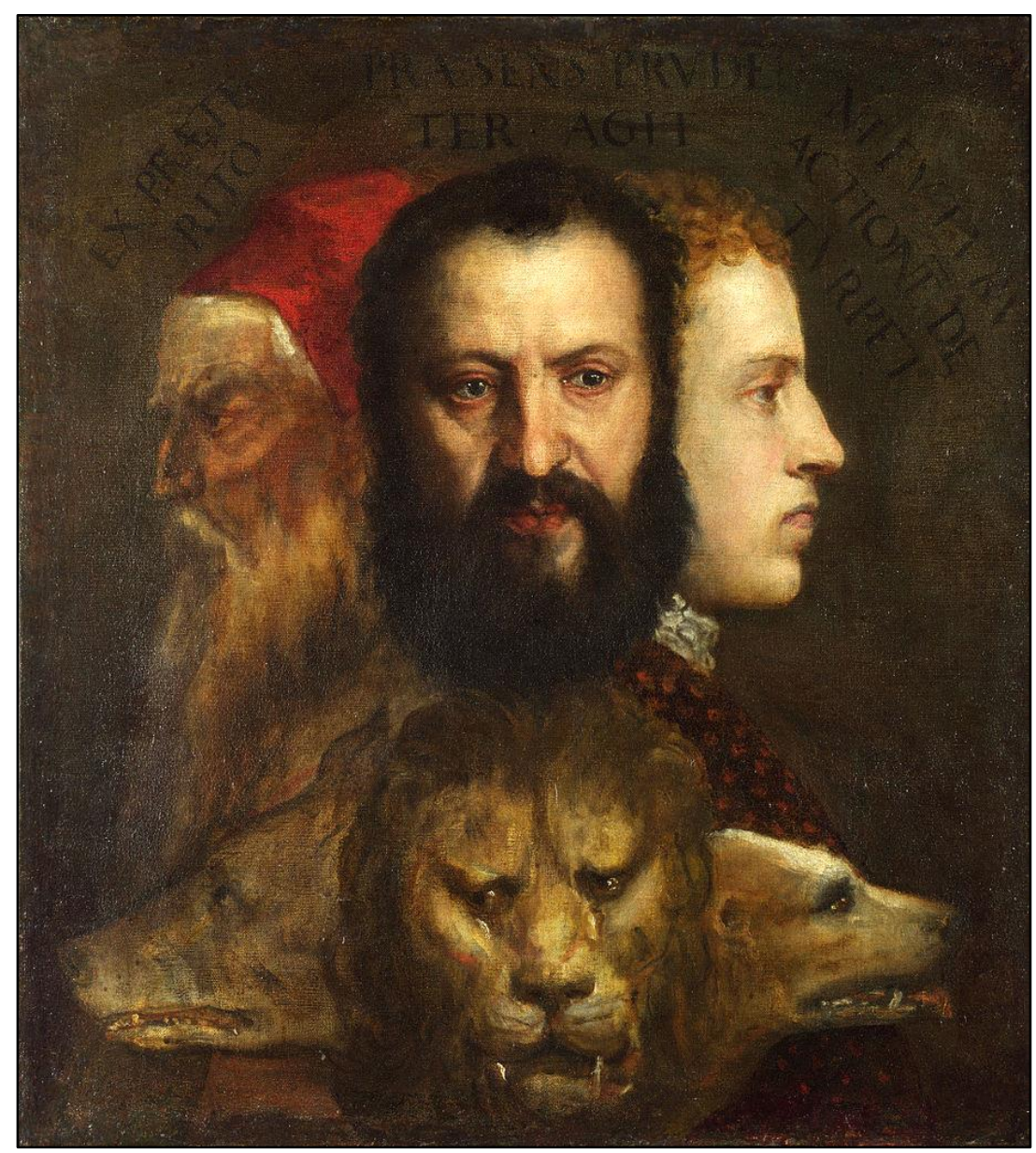

Figura 12: Tiziano, Allegoria della Prudenza, 1565-1570, National Gallery, London.

Ma vorrei notare che la complessa simbologia del quadro non ci distrae da quella luce che si affievolisce progressivamente da destra a sinistra: la luce va dal volto imberbe illuminato, alla fronte volitiva e in piena luce dell'adulto, al volto che sembra dissolversi nell'ombra in cui è immersa la vecchiaia, ombra ribadita dalla barba, quasi uno schermo-tenda. Al di là dei livelli di lettura, colpisce la carica riflessiva e autoriflessiva dei tre ritratti, che non ci guardano, ma sembrano tutti, anche il giovane, immersi nel pensiero del tempo. 
Guardiamo poi la vecchia di Giorgione: si affaccia dal buio a un parapetto, come sull'abisso del tempo; il suo sguardo è rivolto a noi, mentre è concentrata in un gesto autoriflessivo, se non autoaccusatorio, della mano; la bocca aperta sembra pronunciare le parole scritte nel cartiglio che ha in mano: "COL TEMPO”.

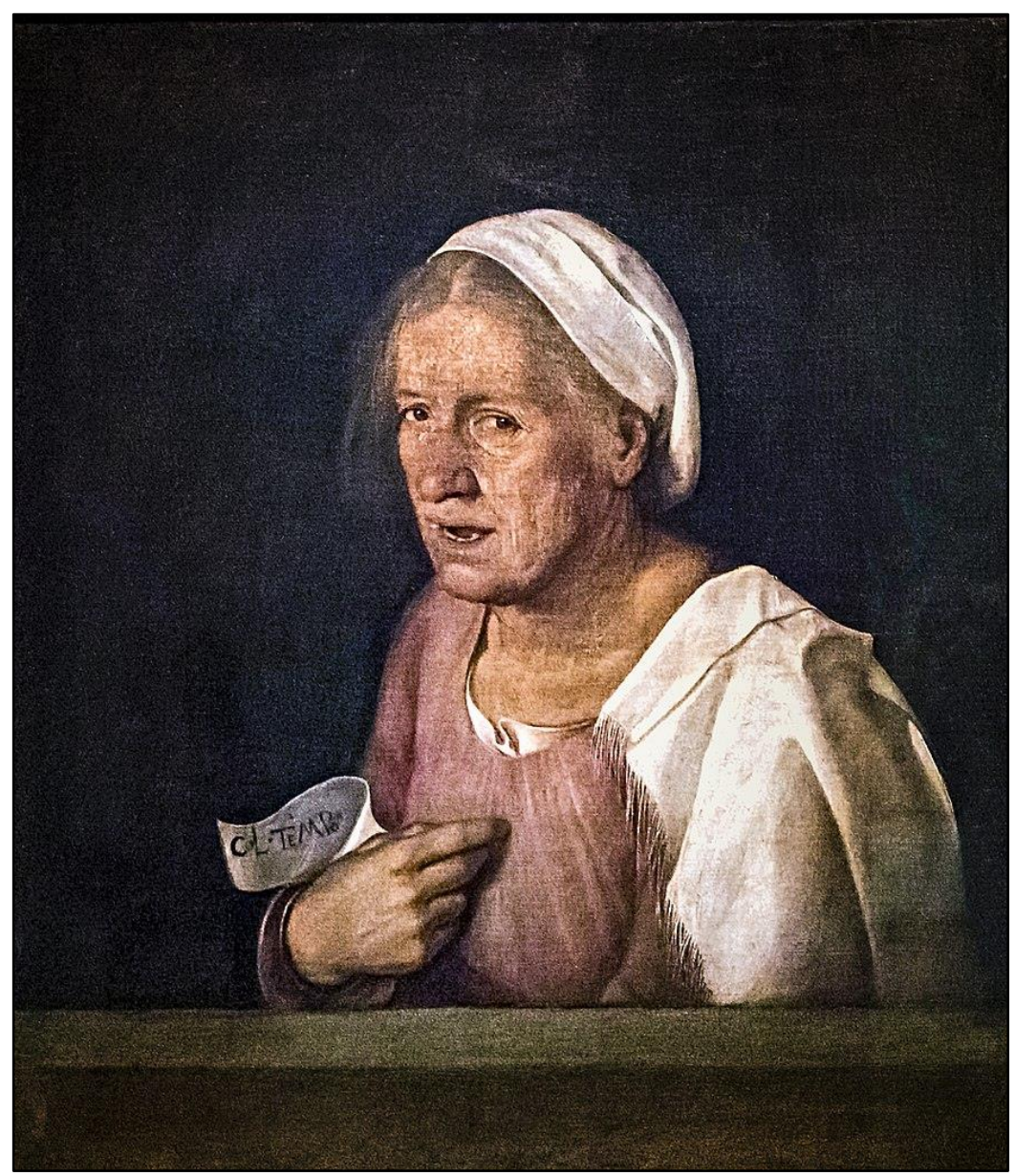

Figura 13: Giorgione, Ritratto di vecchia, 1506 circa, Gallerie dell'Accademia, Venezia.

Guardiamo poi qualche autoritratto di Rembrandt: sappiamo che ne ha dipinti circa settanta. Un esercizio su di sé e sulla propria identità nel tempo che è durato tutta la vita, e in cui ha anche sperimentato finzione e travestimenti fantasiosi (ad esempio, da cavaliere, prelato, studioso, mendicante). Qui consideriamo ritratti che sembrano proporci uno sguardo 
interiore, che si volge alla propria identità con uno sforzo di autoriflessione, e con un notevole scavo psicologico ${ }^{25}$.

Nell'Autoritratto giovanile (prestato dal Rijksmuseum di Amsterdam all'Accademia Carrara di Bergamo per la mostra Rembrandt in una storia meravigliosa, luglio-novembre 2021), un volto di fanciullo, probabilmente a quel tempo apprendista di bottega, ci mostra una guancia morbida e una testa emergente dal buio grazie a una lama di luce.

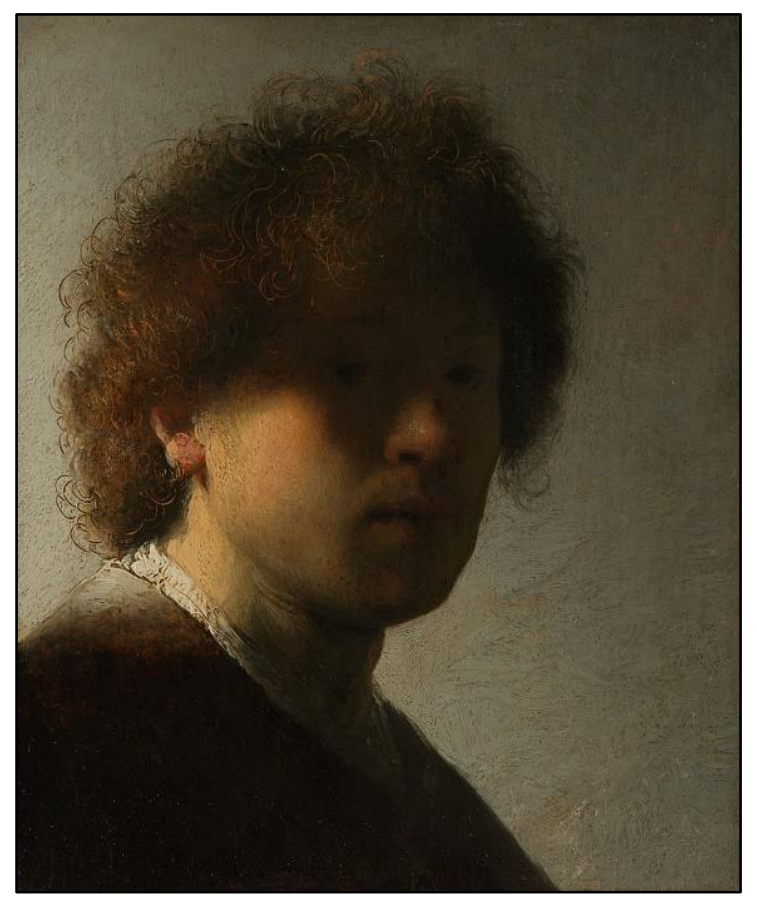

Figura 14: Rembrandt, Autoritratto giovanile, 1628 circa, Rijksmuseum, Amsterdam.

Accostiamo ora tre autoritratti che possiamo guardare come una narrazione. L'autoritratto giovanile ci guarda come dall'alto della giovane età; nei due pensosi autoritratti tardi, notiamo l'approfondirsi dello sguardo, uno sguardo alla fine quasi spossato, ma sempre persuaso di sé.

${ }^{25}$ Cfr. F. Bianco, "Intorno al valore autobiografico della pittura", Oltrecorrente, III, 2001, pp. $51-65$. 


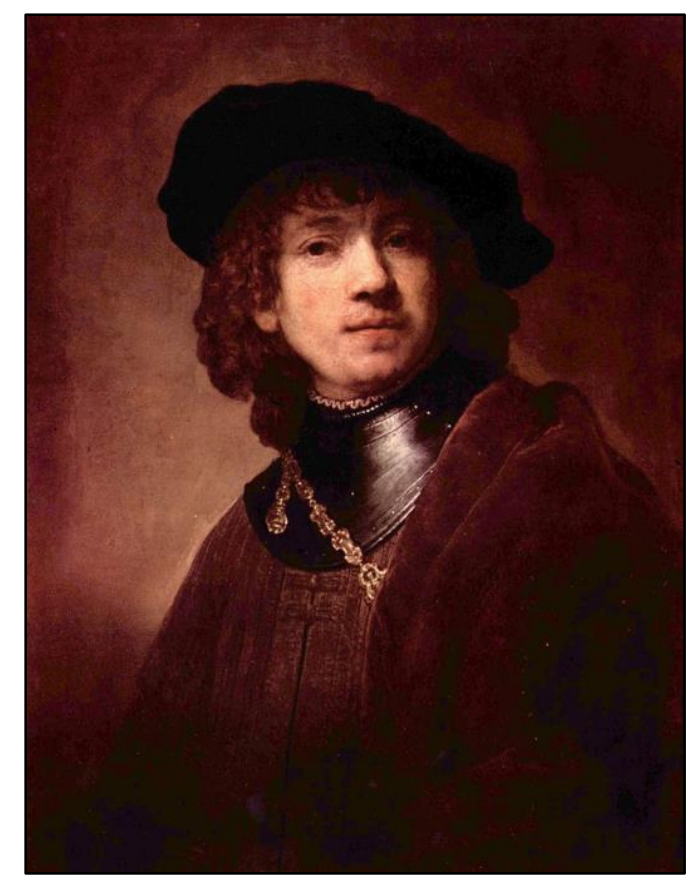

Figura 15: Rembrandt, Autoritratto giovanile, 1634, Galleria degli Uffizi, Firenze.

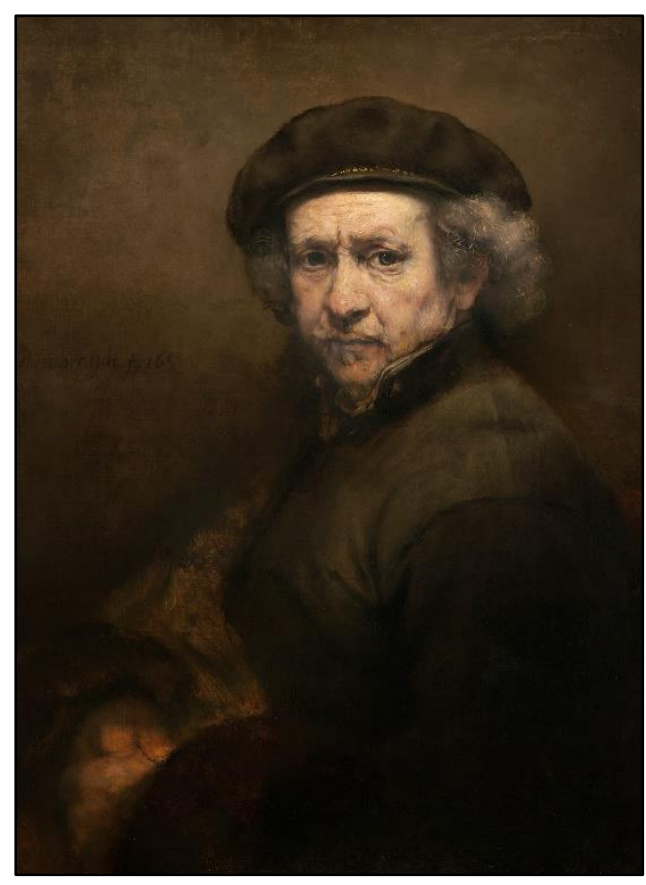

Figura 16: Rembrandt, Autoritratto con berretto e il bavero rialzato, 1659, National Gallery of Art, Washington D.C.

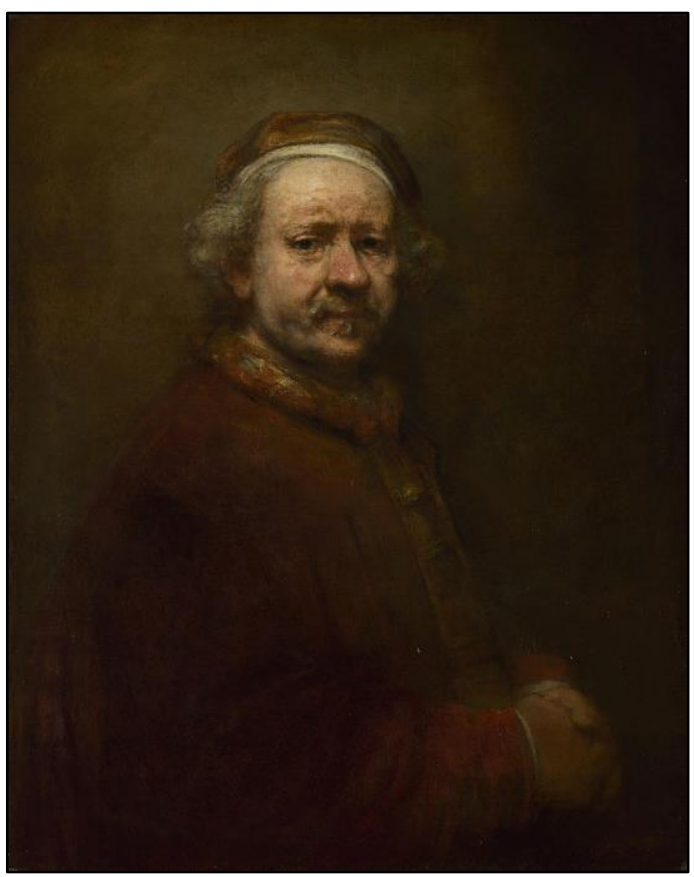

Figura 17: Rembrandt, Autoritratto, 1669, National Gallery, London. 
Proviamo a guardare ancora i ritratti di Rembrandt, ma dal punto di vista della fine, da destra a sinistra. Rovesciare la narrazione ci aiuta a vedere lo sforzo di autocomprensione del pittore nel momento in cui si riducono le possibilità di vita. È quanto suggerisce Marguerite Yourcenar, che scrive le Memorie di Adriano dal punto di vista della morte imminente dell'imperatore:

Un critico ha osservato che i personaggi dei miei libri sono presentati di preferenza nella prospettiva della morte imminente, e che questa toglie ogni significato alla vita. Ma ogni vita è significativa, perfino quella di un insetto, e il sentimento della sua importanza, immensa per chi l'ha vissuta, o almeno quello della sua unicità, aumenta invece di diminuire quando si è vista la parabola concludersi $\mathrm{o}$, in casi più rari, l'iperbole fiammeggiante descrivere la sua curva $\mathrm{e}$ scendere sotto l'orizzonte ${ }^{26}$.

Guardare dal punto di vista della fine può restituire il senso dell'unicità di quel "per sé", e la qualità del suo tempo.

Gli sguardi che abbiamo guardato e che ci hanno guardato in questi ritratti sono sguardi introflessi, come chiedeva Leonardo nel passo del Trattato della pittura che abbiamo letto. Ciò che si dà a vedere nel volto, e che ci guarda, sembra portare alla superficie l'invisibile, cioè l'uomo interiore. Intendo dire con ciò che il ritratto porta alla superficie il tempo dellindividuo non come istante oggettivato, ma come esperienza e come processo che trasforma.

Leonardo parla di questa forza spirituale di cambiamento anche da un altro punto di vista, come se fosse il riflesso del mondo o del tempo cosmico irreversibile sui volti:

Forza dico essere una virtù spirituale, una potenza invisibile $[. .$.$] costrigne tutte$ le cose create a mutazione di forma e sito; corre con furia alla sua desiderata morte e vassi diversificando mediante le cagioni. [...] Scaccia con furia ciò che si oppone a sua soddisfazione, desidera vincere, occidere la sua cagione, il suo contrasto, e, vincendo, se stessa occide; fassi più potente dove trova maggior contrasto 27 .

${ }_{26}$ M. Yourcenar, Care memorie (1974), tr. it. di G. Cillario, Einaudi, Torino 1981, poi Mondadori, Milano 1999, p. 110.

${ }^{27}$ Leonardo, Manoscritto B dell'Institut de France, f. 63r, citato in F. Caroli, "Cinque secoli di pittura verso il profondo", in L'anima e il volto, cit., p. 21. 
Una declinazione brutale e tragica di questa "virtù spirituale" trasformativa e inarrestabile di cui parla Leonardo l'abbiamo riconosciuta nei volti maciullati di Francis Bacon, e nella sua pittura, che egli concepiva come gesto del continuum corpo-mente, come se la pittura fosse stenografia delle sensazioni ${ }^{28}$.

Ancora, possiamo vedere una declinazione beffarda di questa virtù spirituale trasformativa in quei misteriosi segni che Savinio rappresenta nei volti di Padre e Figlio, tuttavia quasi contraddicendosi attraverso l'affettuosa inclinazione e l'accostarsi delle teste.

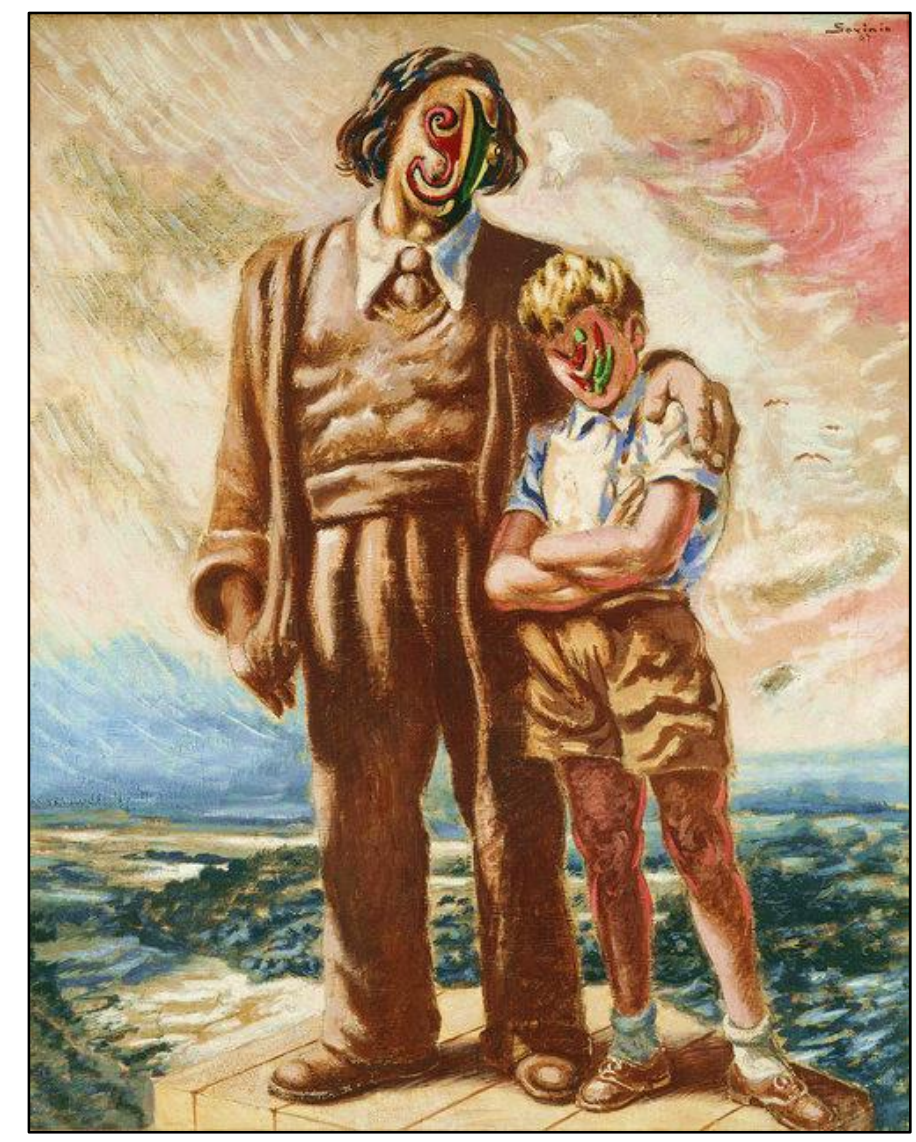

Figura 18: Alberto Savinio, Padre e figlio, 1947,

Collezione privata.

${ }^{28}$ Bacon dichiarava nelle interviste: «Dipingere è proiettare sulla tela il disegno del proprio sistema nervoso". R. Alley, "Francis Bacon e l'arte del ventesimo secolo", in R. Chiappini (a cura di), Francis Bacon, Milano, Electa, 1993, p. 22. 
Savinio, che scrive dell'infanzia come di un tempo mitico, fa qui beffardamente segno all'invecchiamento come tragedia dell'infanzia, ragionando sull'irreversibilità del processo di «sterilizzazione dell'individuo»:

Si immagina un uomo la cui vita sia lo sviluppo naturale, conseguente dell'infanzia? Una siffatta eventualità spaventa l'adulto [...] il potere esecutivo dell'adultità recide l'infanzia: oltre a tutto, la vestizione della toga virile sanziona, con una cerimonia "ambita", l'inizio del "passo ridotto". Un dovere illusorio e la solenne buffoneria della serietà mascherano la tristezza modificata di questo passaggio dal giardino alla cella, dalla libertà al "dovere 29 .

Una declinazione favolistica di questa virtù spirituale trasformativa la troviamo in un affresco di Pisanello.

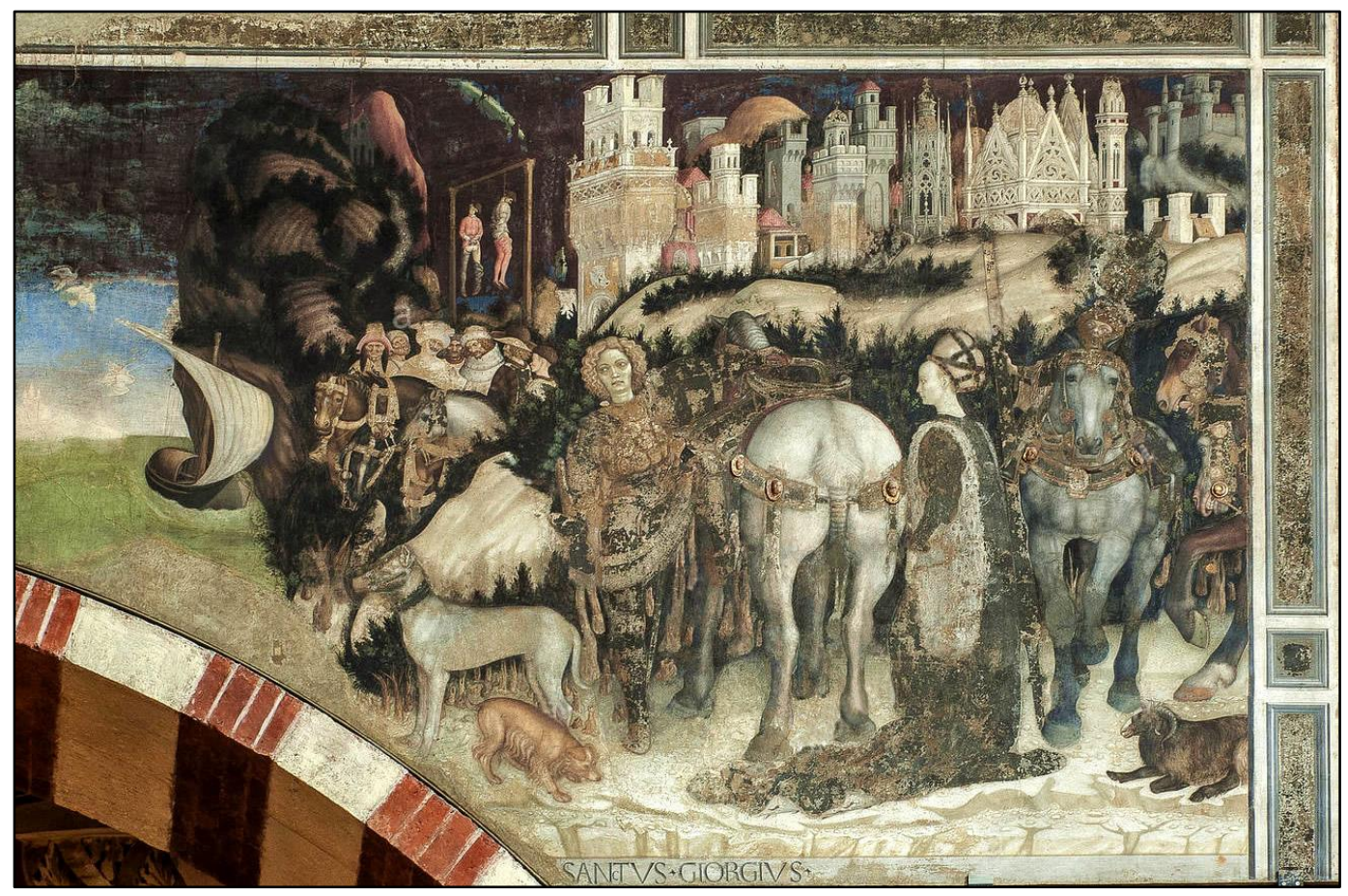

Figura 19: Pisanello, San Giorgio e la Principessa, 1436-1438,

Chiesa di Sant'Anastasia, Verona.

${ }^{29}$ A. Savinio, "Commento alla Tragedia dell'infanzia", in A. Savinio, Tragedia dell'infanzia (1945), tr. it. a cura di P. Italia, Adelphi, Milano 2001, p. 127. 
L'affresco rappresenta San Giorgio che si congeda dalla principessa accingendosi all'impresa in cui affronterà il drago. La scena porta in luce l'alleanza segreta espressa attraverso gli sguardi maschile e femminile: l'eroe parte per l'impresa con il viatico della principessa. Quello che si narra nell'affresco si mostra attraverso la vicenda degli sguardi, che portano alla luce un racconto. W. G. Sebald, in Vertigini, dà una lettura particolarmente significativa del tempo del cambiamento riflesso negli sguardi. Scrive Sebald:

È stupefacente come il Pisanello abbia saputo stabilire un contrasto fra lo sguardo maschile del cavaliere, che si leva all'improvviso e già inclina alla difficile e cruenta impresa, e la risolutezza, unicamente accennata dall'impercettibile abbassarsi dell'orlo inferiore della palpebra, che anima l'occhio femminile ${ }^{30}$.

Sguardi che ci raggiungono nella nostra invisibilità, sguardi che narrano. Nel film Francofonia, il regista Alexander Sokurov si riferisce con queste parole ai ritratti del Louvre: "Chi sarei stato se non avessi potuto vedere gli occhi di chi è vissuto prima di me?».

\section{Nota bibliografica}

ALLEY, Ronald, "Francis Bacon e l'arte del ventesimo secolo", in R. Chiappini (a cura di), Francis Bacon, Milano, Electa, 1993, pp. 15-30.

BIANCO, Franco, "Intorno al valore autobiografico della pittura", Oltrecorrente, III, 2001, pp. 51-65.

CALASSO, Roberto, La folie Baudelaire, Adelphi, Milano 2008.

CAROLI, Flavio, "Cinque secoli di pittura verso il profondo", in F. Caroli, L’anima e il volto. Ritratto e fisiognomica da Leonardo a Bacon, Electa, Milano 1998, pp. 19-28.

${ }^{30}$ W. G. Sebald, Vertigini (1995), tr. it. di A. Vigliani, Adelphi, Milano 20034, pp. 73-74. 
—, "I moti dell'animo", in F. Caroli, L'anima e il volto. Ritratto e fisiognomica da Leonardo a Bacon, Electa, Milano 1998, pp. 39-518.

DAVAL, Jean-Luc, “Fou de réalité. Alberto Giacometti”, Journal de Genève, 8 giugno 1963.

GARELLI, Jacques, "Voir ceci et voir selon", in M. Richir e. Tassin (éd. par), Merleau-Ponty. Phénoménologie et expériences, Millon, Grenoble 1992, pp. 79-99.

GOODMAN, Nelson, I linguaggi dell'arte (1968), tr. it. a cura di F. Brioschi, il Saggiatore, Milano 1976.

HEIDEGGER, Martin, "L'origine dell'opera d'arte", in Sentieri interrotti (1950), tr. it. a cura di P. Chiodi, La nuova Italia, Firenze 1968, pp. 3-69.

HUSSERL, Edmund, Meditazioni cartesiane (1950), tr. it. a cura di F. Costa, Bompiani, Milano 1960.

LAVAUD, Laurent, L’image, Flammarion, Paris 1999.

LEONARDO DA VINCI, Trattato della pittura [on-line]. Edizione digitale a cura di A. Borzelli, tratta da "Trattato della pittura" di Leonardo da Vinci, Carabba Editore, Lanciano 1947. Pubblicato in data 17 febbraio 2016, consultato in data 15 novembre 2021. Disponibile all'indirizzo: https://www.liberliber.it/mediateca/libri///leonardo/trattato_della_pittura/ html/index.htm.

—, I Manoscritti dell'Institut de France, edizione in facsimile, Giunti, Firenze 1986.

MARTINI, Alberto, Alberto Giacometti, in I maestri del colore, n. 79, Fratelli Fabbri, Milano 1965. 
MERLEAU-PONTY, Maurice, L'occhio e lo spirito (1964), tr. it. di A. Sordini, SE, Milano 1989.

—, Il visibile e l'invisibile (1964), nuova ed. it. a cura di M. Carbone, Bompiani, Milano 1993.

—, La prosa del mondo (1969), tr. it. a cura di C. Sini, Editori Riuniti, Roma 1984.

NANCY, Jean-Luc, Il ritratto e il suo sguardo (2000), tr. it. a cura di R. Kirchmayr, Raffaello Cortina, Milano 2002.

PETRELLA, Fausto, "Quadro e cornice: il setting clinico", in F. Petrella, Turbamenti affettivi e alterazione dell'esperienza, Raffaello Cortina, Milano 1993, pp. 127-141.

SAVINIO, Alberto, "Commento alla Tragedia dell'infanzia", in A. Savinio, Tragedia dellinfanzia (1945), tr. it. a cura di P. Italia, Adelphi, Milano 2001, pp. 123-130.

—, "La figura e l'altro", in Quaderni Warburg Italia, I, 2003, pp. 319-328.

SEBALD, W. G., Vertigini (1995), tr. it. di A. Vigliani Adelphi, Milano $2003^{4}$.

SOLDINI, Jean, Alberto Giacometti. La somiglianza introvabile, Jaca Book, Milano 1998.

TADINI, Emilio, "Il santo, il dialogo", in G. Damiani (a cura di), L’immagine. Arte, scienza, teoria, Carlini, Milano 1989, pp. 205-214.

WITTGENSTEIN, Ludwig, Ricerche filosofiche (1953), tr. it. e cura di M. Trinchero, Einaudi, Torino 1967, ristampa 1974. 
—, Osservazioni sui colori (1977), tr. it. di M. Trinchero, introduzione di A. G. Gargani, Einaudi, Torino 1982

—, Osservazioni sulla filosofia della psicologia (1980), tr. it. a cura di R. De Monticelli, Adelphi, Milano 1990.

YOURCENAR, Marguerite, Care memorie (1974), tr. it. di G. Cillario, Einaudi, Torino 1981, poi Mondadori, Milano 1999.

\section{Nota biografica}

Già professore ordinario di Filosofia teoretica all’Università di Pavia, continua la sua ricerca nel campo delle scienze umane, delle teorie dell'immagine e della traduzione. Tra le pubblicazioni: Filosofia dei sensi. Estetica del pensiero, tra filosofia, arte e letteratura (Cortina, Milano 2006); Leggere il "Tractatus logico-philosophicus» di Wittgenstein (Ibis, Como-Pavia 2010); con U. Heidmann, La Babele in cui viviamo. Traduzioni, riscritture, culture (Bollati Boringhieri, Torino 2012); Nodi della verità. Concetti e strumenti per le scienze umane (Mimesis, Milano 2017)); con L. Vanzago, Dubitare, riflettere, argomentare. Percorsi di filosofia teoretica (Carocci, Roma 2018). 